\title{
Spontaneous Neural Oscillations Bias Perception by Modulating Baseline Excitability
}

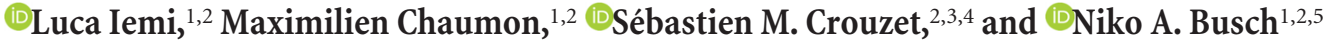 \\ ${ }^{1}$ Berlin School of Mind and Brain, Humboldt-Universität zu Berlin, 10099 Berlin, Germany, ${ }^{2}$ Institute of Medical Psychology, \\ Charité-Universitätsmedizin, 10117 Berlin, Germany, ${ }^{3}$ Université de Toulouse, UPS, Centre de Recherche Cerveau et Cognition, 31062 Toulouse, France, ${ }^{4}$ CerCo, \\ CNRS UMR 5549, 31062 Toulouse, France, and Institute of Psychology, Westfälische Wilhelms-Universität Münster, 48149 Münster, Germany
}

The brain exhibits organized fluctuations of neural activity, even in the absence of tasks or sensory input. A prominent type of such spontaneous activity is the alpha rhythm, which influences perception and interacts with other ongoing neural activity. It is currently hypothesized that states of decreased prestimulus $\alpha$ oscillations indicate enhanced neural excitability, resulting in improved perceptual acuity. Nevertheless, it remains debated how changes in excitability manifest at the behavioral level in perceptual tasks. We addressed this issue by comparing two alternative models describing the effect of spontaneous $\alpha$ power on signal detection. The first model assumes that decreased $\alpha$ power increases baseline excitability, amplifying the response to both signal and noise, predicting a liberal detection criterion with no effect on sensitivity. The second model predicts that decreased $\alpha$ power increases the trial-by-trial precision of the sensory response, resulting in improved sensitivity. We tested these models in two EEG experiments in humans where we analyzed the effects of prestimulus $\alpha$ power on visual detection and discrimination using a signal detection framework. Both experiments provide strong evidence that decreased $\alpha$ power reflects a more liberal detection criterion, rather than improved sensitivity, consistent with the baseline model. In other words, when the task requires detecting stimulus presence versus absence, reduced $\alpha$ oscillations make observers more likely to report the stimulus regardless of actual stimulus presence. Contrary to previous interpretations, these results suggest that states of decreased $\alpha$ oscillations increase the global baseline excitability of sensory systems without affecting perceptual acuity.

Key words: alpha rhythm; excitability; neural oscillations; perception; signal detection theory; spontaneous activity

Significance Statement

Spontaneous fluctuations of brain activity explain why a faint sensory stimulus is sometimes perceived and sometimes not. The prevailing view is that heightened neural excitability, indexed by decreased $\alpha$ oscillations, promotes better perceptual performance. Here, we provide evidence that heightened neural excitability instead reflects a state of biased perception, during which a person is more likely to see a stimulus, whether or not it is actually present. Therefore, we propose that changes in neural excitability leave the precision of sensory processing unaffected. These results establish the link between spontaneous brain activity and the variability in human perception.

\section{Introduction}

The brain exhibits organized fluctuations of neural activity, even in the absence of tasks or sensory input. This activity is referred

Received May 1, 2016; revised Nov. 16, 2016; accepted Dec. 1, 2016.

Author contributions: L.I., M.C., and N.A.B. designed research; L.I. performed research; L.I., M.C., S.M.C., and N.A.B. contributed unpublished reagents/analytic tools; L.I. and N.A.B. analyzed data; L.I., M.C., and N.A.B. wrote the paper.

This work was supported by the German Academic Exchange Service DAAD to L.I., the Berlin School of Mind and Brain to L.I., and Deutsche Forschungsgemeinschaft Research Grant CH1246-1 to M.C. We thank Lena Walther for assistance with the EEG recording and preprocessing and Rasa Gulbinaite for helpful comments and suggestions on an earlier version of the manuscript.

The authors declare no competing financial interests.

Correspondence should be addressed to Dr. Luca lemi, Berlin School of Mind and Brain, Humboldt-Universität zu Berlin, Unter den Linden 6, 10099 Berlin, Germany. E-mail: luca.iemi@gmail.com.

DOI:10.1523/JNEUROSCI.1432-16.2016

Copyright $\odot 2017$ the authors $\quad 0270-6474 / 17 / 370807-13 \$ 15.00 / 0$ to as "ongoing", "spontaneous", or "prestimulus", as opposed to stimulus-evoked or experimentally induced. A number of studies have demonstrated that spontaneous oscillations in the $\alpha$ frequency band $(8-12 \mathrm{~Hz})$ persist during task performance and have substantial perceptual and neural effects. Specifically, states of decreased $\alpha$ power are thought to reflect a state of enhanced neural excitability (Mazaheri and Jensen, 2010; Harvey et al., 2013; van Kerkoerle et al., 2014), which in turn is supposed to facilitate stimulus perception (Ergenoglu et al., 2004; Romei et al., 2008; van Dijk et al., 2008; Roberts et al., 2014; Baumgarten et al., 2016). However, the mechanisms underlying this facilitation are currently not well understood, and the evidence for an effect of prestimulus $\alpha$ power on perceptual performance appears to be inconsistent (for a comprehensive literature review, see Table 1). Clarifying how changes in neuronal excitability are related to 
Table 1. Literature review on the effect of $\alpha$ power on behavioral performance in visual tasks ${ }^{a}$

\begin{tabular}{|c|c|c|c|c|}
\hline & Reference & Paradigm & Stimulus absent trials $(\%)$ & $\alpha$ power \\
\hline \multirow[t]{16}{*}{ Affected by baseline shifts } & Boncompte et al. (2016) & Detection of stimulus presence/absence & 15 & $\mathrm{H}<\mathrm{M}^{b}$ \\
\hline & Limbach and Corballis (2016) & Detection of stimulus presence/absence & 50 & $\mathrm{H}<\mathrm{M} ; \mathrm{FA}<\mathrm{CR}$ \\
\hline & Britz et al. (2014) & Report awareness of the stimulus & 0 & $H=M$ \\
\hline & Chaumon and Busch (2014) & Detection of stimulus presence & 0 & $H<M$ \\
\hline & Mathewson et al. (2014) & Detection of stimulus presence/absence & 25 & $H<M$ \\
\hline & Roberts et al. (2014) & Detection of target presence within a series of nontargets & 80 & $H<M$ \\
\hline & Keil et al. (2014) & Double-flash illusion & 65 & $\mathrm{FA}<\mathrm{CR}^{b}$ \\
\hline & Lange et al. (2013) & Fusion effect; double-flash illusion & 20 & $\mathrm{H}<\mathrm{M} ; \mathrm{FA}<\mathrm{CR}$ \\
\hline & Achim et al. (2013) & Detection of stimulus presence & 0 & $\mathrm{H}<\mathrm{M}$ \\
\hline & Busch and VanRullen (2010) & Detection of stimulus presence & 11 & $H<M$ \\
\hline & Busch et al. (2009) & Detection of stimulus presence/absence & 20 & $\mathrm{H}<\mathrm{M}$ \\
\hline & Mathewson et al. (2009) & Detection of stimulus presence/absence & 25 & $H<M$ \\
\hline & Romei et al. (2008) & Report awareness of the phosphene & 0 & $H<M$ \\
\hline & van Dijk et al. (2008) & Detection of the presence/absence of a contrast difference & 26 & $H<M$ \\
\hline & Babiloni et al. (2006) & Detection of stimulus presence/absence & 33.3 & $\mathrm{H}>\mathrm{M}$ \\
\hline & Ergenoglu et al. (2004) & Detection of stimulus presence & 0 & $H<M$ \\
\hline \multirow[t]{6}{*}{ Unaffected by baseline shifts } & Limbach and Corballis (2016) & Detection of stimulus presence/absence & 50 & $C=\mathbb{I N}$ \\
\hline & Bays et al. (2015) & 2AFC discrimination & - & $\mathrm{C}=\mathrm{IN}$ \\
\hline & Lou et al. (2014) & 2AFC discrimination & - & $C<\mathbb{N}$ \\
\hline & Wutz et al. (2014) & 5 AFC discrimination & - & $C=\mathbb{I N}$ \\
\hline & Macdonald et al. (2011) & Detection of stimulus presence/absence & 50 & $C=\mathbb{N}$ \\
\hline & Hanslmayr et al. (2007) & 4AFC discrimination & - & $\mathrm{C}=\mathbb{I N}$ \\
\hline
\end{tabular}

${ }^{a}$ The majority of studies analyzing performance measures, which are affected by changes in baseline excitability/criterion, report decreased $\alpha$ power preceding "yes" reports (H $<\mathrm{M}$, $\mathrm{FA}<\mathrm{CR}$; top). By contrast, the majority of studies analyzing performance measures, which are unaffected by changes in baseline excitability/criterion, report null effects $(C=I N$; bottom). Experimental effects are indicated by comparing $\alpha$ power $(=,<,>)$ between different response types: $\mathrm{H}$, Hits; $\mathrm{M}$, misses; FA, false alarms; $\mathrm{CR}$, correct rejections; $\mathrm{C}$, correct responses; IN, incorrect responses. AFC, alternative forced choice.

${ }^{b}$ Nonsignificant trend.

changes in performance addresses the fundamental question of how the momentary internal state of a neural system interacts with input from the external world.

The key to understanding how and under which conditions $\alpha$ oscillations affect perceptual performance is to relate fluctuations of $\alpha$ power to changes in perceptual decision making using a formal model. Here, we formulated two alternative models of this relationship using signal detection theory (SDT) (Green and Swets, 1966).

In the baseline model (Fig. 1, left), decreases in $\alpha$ power are hypothesized to increase the global baseline excitability, thereby changing the positions of both signal and noise distributions with respect to the criterion $(k)$. This model is based on the observed negative relationship between $\alpha$ power and baseline neural activity (Haegens et al., 2011; Harvey et al., 2013; van Kerkoerle et al., 2014). In a yes/no signal detection paradigm, increased baseline excitability corresponds to a more liberal detection criterion (i.e., increased tendency to make "yes" reports regardless of actual stimulus presence) (Fig. 1, top left). By contrast, this model predicts no effect on behavior in multiple alternative forced choice (mAFC) tasks. Indeed, while detection decisions are made by comparing the internal response, $R$, with $k$ ("yes" if $R>k$; otherwise, "no"), 2AFC discrimination decisions are made by comparing the responses of two signal detectors $R_{1}$ and $R_{2}$ (" $\mathrm{A}$ " if $R_{1}>R_{2}$; otherwise, "B"). Accordingly, a baseline shift would
Baseline Excitability
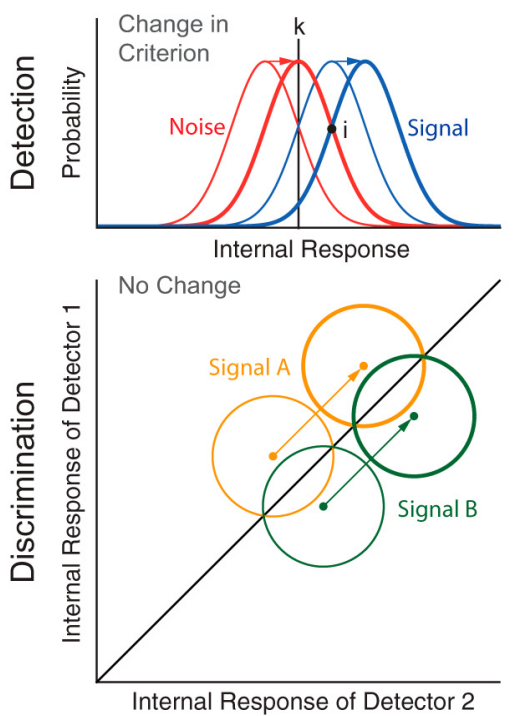

Figure 1. Signal detection models. The perceptual effect of $\alpha$ oscillations can be modeled in terms of SDT. In SDT, sensitivity reflects the distance between the means of the internal distributions, divided by their common SD. In yes/no detection task, criterion reflects the location of the decision criterion $k$ relative to the point of intersection between the internal distributions $i$. According to the baseline excitability model, reduced $\alpha$ power enhances the response to both signal and noise, thereby inducing a more liberal criterion in detection tasks (top left) and leaving performance unaffected in discrimination tasks (bottom left). By contrast, the response precision model predicts that reduced $\alpha$ power improves sensitivity in both detection (top right) and discrimination tasks (bottom right) due to reduced trial-by-trial response variability.

\section{Response Precision}
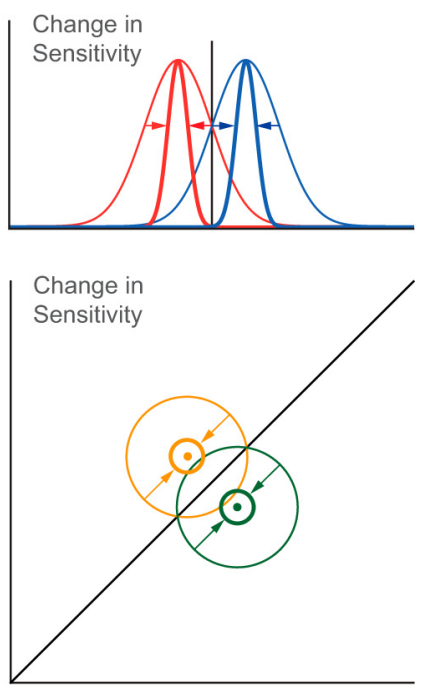

equally affect $R$ of all signal detectors without changing their relative strength, leaving discrimination accuracy unaffected (Fig. 1, bottom left).

In the precision model (Fig. 1, right), decreases in $\alpha$ power are hypothesized to reduce the trial-by-trial variability of the sensory response, thereby increasing the precision of the internal signal 
a

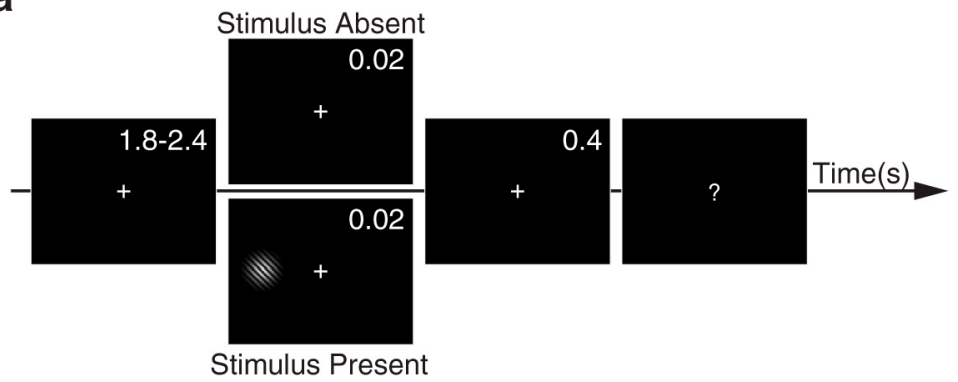

b

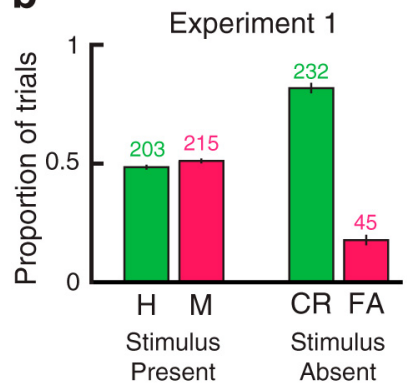

Experiment 2

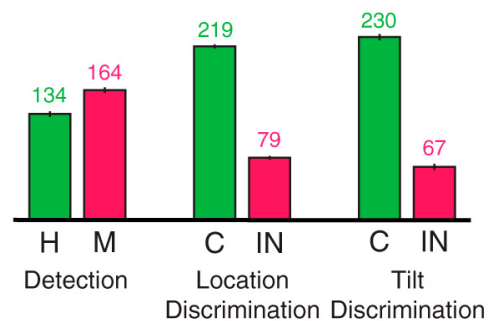

Figure 2. Experimental paradigm and behavior. $\boldsymbol{a}$, In Experiment 1, a near-threshold stimulus was presented for $0.02 \mathrm{~s}$ in $60 \%$ of trials (stimulus present trials). In the remaining $40 \%$ of trials, a blank screen was presented for the same duration (stimulus absent trials). The participants were instructed to perform a yes/no detection task. In Experiment 2, all trials contained a target stimulus. At the beginning of each experimental block, the participants were instructed to perform a yes/no detection, location discrimination, or tilt discrimination task. $\boldsymbol{b}$, Mean proportion of correct ( $\mathrm{H}$, Hits; $\mathrm{CR}$, correct rejections; $\mathrm{C}$, correctly discriminated) and incorrect (M, Misses; FA, false alarms; IN, incorrectly discriminated) responses in Experiments 1 and 2. Error bars indicate SEM. Numbers above bars indicate the median number of trials across participants.

representation. This model is based on studies showing that response precision is increased by attention (Cohen and Maunsell, 2009; Mitchell et al., 2009), which is known to modulate $\alpha$ power. In a yes/no signal detection paradigm, enhanced precision improves perceptual acuity by increasing the separation between signal and noise distributions, and thus the sensitivity parameter (Fig. 1, top right). In mAFC tasks, this model also predicts an increase in discrimination accuracy because increased precision enhances the separation between the response probability distributions of the detectors representing the alternative stimuli (Fig. 1 , bottom right).

We directly tested these models in two EEG experiments by analyzing the effects of $\alpha$ power on sensitivity and criterion in a detection task and by comparing the perceptual effects of $\alpha$ oscillations in detection and discrimination tasks. Both experiments provided strong evidence for the baseline model: reduced $\alpha$ power reflected a more liberal criterion, rather than improved perceptual acuity in both detection and discrimination tasks. These findings largely resolve inconsistencies in previous literature and specify the link between spontaneous $\alpha$ oscillations, neural excitability, and performance in perceptual tasks.

\section{Materials and Methods}

\section{Participants}

Sixty-eight participants with normal or corrected-to-normal vision and no history of neurological disorders took part in this study. In Experiment 1, 33 participants were tested (mean \pm SEM: $27.7 \pm 0.59$ years, 19 females, 7 left-handed). Two participants were excluded before EEG preprocessing because they performed at chance level (see Behavioral exclusion criteria). One participant was excluded after EEG preprocessing because of excessive artifacts. A total of 30 participants were included in the analyses for Experiment 1. In Experiment 2, 35 participants were tested (mean \pm SEM: $27.5 \pm 0.61$ years, 19 females, 5 left-handed). Four participants were excluded before EEG preprocessing because of poor behavioral performance (see Behavioral exclusion criteria). Five participants were excluded after EEG preprocessing because of excessive artifacts. A total of 26 participants were included in the analyses for Experiment 2. Before both experiments, written informed consent was obtained from all participants. All experimental procedures were approved by the ethics committee of the German Psychological Society.

\section{Stimuli}

Both experiments were written in MATLAB (RRID: SCR_001622) using the Psychophysics Toolbox 3 (RRID: SCR_ 002881, Brainard, 1997; Pelli, 1997). The stimuli were presented on a black background using a cathode ray tube monitor operated at $100 \mathrm{~Hz}$ and situated in a dark room. Target stimuli consisted of lowcontrast Gabor patches tilted 10 degrees, and with a diameter of 0.75 degrees of visual angle. The stimuli were displayed at 10 degrees of visual angle to the left or to the right of the fixation cross for a duration of two frames $(0.02 \mathrm{~s})$. For both experiments, stimulus side and tilt were counterbalanced. In Experiment 1, the target stimulus was present in $60 \%$ of the trials and absent in $40 \%$ of the trials, during which a blank screen was presented for a duration of two frames (0.02 s; Fig. 2a). In Experiment 2, a stimulus was present in all trials. After a delay of $400 \mathrm{~ms}$ following target offset, the fixation cross turned into a question mark, which instructed the participants to deliver a response via button-press, in accordance with the task instructions. After this, the fixation cross was displayed again and a new trial started. The following target stimulus was presented after a variable delay chosen from a uniform distribution between 1.8 and $2.4 \mathrm{~s}$.

\section{Behavioral paradigm}

Experiment 1 was designed to examine the effect of prestimulus power on yes/no detection behavior. Here, participants were asked to report whether or not they perceived the target stimulus. For each participant, an adaptive staircase procedure (QUEST) (Watson and Pelli, 1983), was used to find a stimulus contrast yielding a hit rate $(H R)$ of $50 \%$ (proportion of "yes" reports in stimulus present trials). The participants were instructed that some trials contained a stimulus and others did not. Immediately after the detection task, the participants were required to rate the confidence of their previous response on a 3-point scale. At the end of each trial, participants received color-coded feedback on the detection report. Experiment 1 included 700 trials divided into 14 blocks of 50 trials each.

Experiment 2 was designed to compare the effect of prestimulus power on performance between a yes/no detection task and discrimination tasks of equal task difficulty. This comparison precluded the use of stimulus absent trials for two reasons. First, it is not possible to estimate whether a discrimination report (stimulus " $\mathrm{A}$ " or " $\mathrm{B}$ ") is accurate when no stimulus is presented, precluding the use of stimulus absent trials in discrimination tasks. Second, including stimulus absent trials only in the yes/no detection task, but not in the discrimination tasks, would have confounded any comparison between tasks. In other words, any potential differences between tasks could have been attributed to this difference in the procedure instead of a difference in perceptual processing. Because Experiment 2 forbids the use of stimulus absent trials, signal detection measures could not be estimated. Experiment 2 included 900 trials divided into 18 blocks of 50 trials each. The blocks were presented in a randomized order.

For each participant, a QUEST was used to determine on each trial the target contrast level to achieve a $H R$ of $50 \%$ for the detection task and a 
proportion of correct responses of $75 \%$ in the discrimination tasks. These different values between detection and discrimination tasks were necessary to ensure equal difficulty across tasks, such that the stimulus was perceived approximately on every second trial. Indeed, in a yes/no detection task, the $H R$ of a blindfolded participant who reports truthfully her perception (or lack thereof) is expected to be $0 \%$, whereas the $H R$ of a participant that sees the stimulus on every other stimulus-present trial is $50 \%$ (assuming that the participant is not substantially biased); contrariwise, in 2AFC tasks, the proportion of correct responses of a blindfolded participant who is guessing is $50 \%$, whereas the proportion of correct responses of a participant that discriminates the stimulus on every other trial is $50 \%$ (i.e., guessing) + $0.5^{\star} 50 \%=75 \%$.

A blocked design was chosen over a trialby-trial design because a trial-by-trial design would require using the same stimulus in all trials, regardless of the task. However, as reported in Results, tilt discrimination required a much higher contrast than detection and localization. Thus, using the same stimulus for all tasks would result in widely different performance levels. Second, a trial-by-trial design also assumes that detection and discrimination are fully independent in terms of decisionmaking. This is, however, unlikely to be the case: when subjects detect that a stimulus was present but cannot discriminate its location or tilt, they are likely to be biased to report the stimulus as absent. As Experiment 2 aimed to test the different effects of neural oscillations on detection and discrimination, it was therefore indispensable to present tasks block-wise.

Notably, the stimulus present trials of Experiment 1 and the detection trials of Experiment 2 were designed to be identical regarding all experimental parameters (i.e., tilt, diameter, duration, eccentricity, intertrial interval, QUEST parameters), and thus can be directly compared between experiments.

In both experiments, to ensure that trials included in the analyses had stimuli with similar contrast, we rejected outlier trials in which the presented contrast value differed from the final threshold estimated by the individual QUEST by more than an arbitrary threshold of 0.2 contrast units. In Experiment 2, this was calculated separately for each task.

Behavioral exclusion criteria. In an experiment with an infinite number of trials, a $d^{\prime}$ of 0 indicates that an observer cannot discriminate a stimulus from noise, whereas an infinite $d^{\prime}$ indicates perfect performance. However, with a finite number of trials, it is possible that an observer with null sensitivity achieves a $d^{\prime}>0$ because of lucky guessing. To account for guessing in Experiment 1, we simulated the performance of an observer with null sensitivity in a detection task with the same number of stimulus present and absent trials as in Experiment 1. A "yes" or a "no" response was randomly drawn for each trial; then $d^{\prime}$ was calculated. This procedure was repeated 10,000 times; and for each participant, a $p$ value was computed as the proportion of the simulated $d^{\prime}$ measures that exceeded the observed $d^{\prime}$. We excluded from EEG analysis 2 participants with a $p$ value of 0.9 and 0.2 , which indicated that task performance was not significantly better than chance.

In Experiment 2, we excluded four participants because the contrast value found by QUEST reached a maximum value of 1 (full contrast) during more than one experimental block, indicating a poor behavioral performance.

\section{EEG recording and preprocessing}

A 64-channels ActiveTwo system (Biosemi) was used to record continuous EEG sampled at $1024 \mathrm{~Hz}$ while the participants performed the experimental tasks. Electrodes were placed according to the international
10-10 system. The horizontal and vertical electro-oculograms were recorded by attaching additional electrodes at the lateral canthi of both eyes and below the eyes, respectively. The EEGLAB toolbox version 11 running on MATLAB (R2010b; The MathWorks) was used to process and analyze the data (Delorme and Makeig, 2004). Data were rereferenced to the mastoids, epoched from -2000 to $1500 \mathrm{~ms}$ relative to target onset, down-sampled to $256 \mathrm{~Hz}$, and bandpass filtered between 0.25 and $80 \mathrm{~Hz}$, as recommended by Widmann et al. (2015). Major artifacts (eye blinks, and noisy data segments) were screened manually, and entire trials were discarded when a blink occurred within a $1 \mathrm{~s}$ time window preceding target onset, to ensure that participant's eyes were open at stimulus onset. On average, we removed 11 (SEM 6.9) and 7 (SEM 2.2) trials in Experiments 1 and 2, respectively. Noisy channels were selected manually for interpolation with the data from the adjacent channels. Furthermore, the EEG data were transformed using independent component analysis, and SASICA (Chaumon et al., 2015) was used to guide the exclusion of independent component related to noisy channels, blinks, eye movements, and muscular contractions. On average, we excluded 8.5 (SEM 0.65) and 11.5 (SEM 0.60) independent components in Experiments 1 and 2, respectively.

\section{Time-frequency analysis}

Time-frequency analysis was performed using a wavelet transform (Morlet wavelets, frequency range: $1-30 \mathrm{~Hz}$, number of cycles increasing linearly from 1 to 12). Thus, a wavelet at $10 \mathrm{~Hz}$ was 4.4 cycles long and had a temporal resolution $\sigma_{\mathrm{t}}$ of $0.14 \mathrm{~s}$ and a spectral resolution $\sigma_{\mathrm{f}}$ of $4.53 \mathrm{~Hz}$. Frequencies and time points were sampled every $2 \mathrm{~Hz}$ and $40 \mathrm{~ms}$, respectively. Because we were primarily interested in the prestimulus time range, no baseline correction was applied.

Because wavelet analysis is computed by convolution of the data with a function that is extended in time, it is conceivable that prestimulus effects close to stimulus onset are actually affected by poststimulus data. To determine the extent of this contamination, we applied a timefrequency transform using the same settings as in the main analysis to synthetic data (i.e., a sinusoidal oscillation of exactly $10 \mathrm{~Hz}$ ) (Fig. 3). The extent of temporal contamination caused by a wavelet is determined by the wavelet's temporal resolution $\sigma_{t}$, which is defined as twice the SD of the Gaussian envelope (Tallon-Baudry et al., 1996). This simulation illustrates that prestimulus time points are indeed contami- 
nated by poststimulus data points. However, the magnitude of this contamination is virtually null at time points earlier than onset: $\sigma_{\text {t }}$. Thus, we consider effects as truly "prestimulus" only if they occur before this limit, which is indicated by a red line in Figures $4-7$. The MATLAB code used for this simulation can be downloaded from https://github.com/LucaIemi/Iemi2017_JNeurosci.

EEG behavior analysis. To analyze how oscillatory power influences performance, we first identified trials with particularly weak and strong power, and then tested how these trials differed in performance measures. Thus, for each time, frequency, and electrode, trials were sorted from weak to strong power and divided into 5 bins. The binning was done separately for each participant (Linkenkaer-Hansen et al., 2004; Lange et al., 2012; Baumgarten et al., 2016).

Experiment 1. We first binned stimulus present and absent trials separately and then calculated the hit rate (HR; the proportion of "yes" reports in signal present trials), false alarm rate (FAR; the proportion of "yes" reports in signal absent trials), as well as signal detection measures of sensitivity and criterion, for each bin and participant. Signal detection measures were estimated following distribution-dependent (Green and Swets, 1966) and distribution-independent (Kornbrot, 2006) SDT analyses.

Distribution-dependent SDT analysis: we estimated measures of sensitivity $\left(d^{\prime}\right)$ and criterion (c) following standard SDT analysis (Green and Swets, 1966). $d^{\prime}$ indicates the ability to discriminate between signal and noise and is represented as the distance between the means of the internal distributions, divided by their common SD (Fig. 1, top right). The higher the sensitivity, the further apart the distributions are in the decision space, the more accurate the performance. Formally, $d^{\prime}$ is computed by subtracting the $z$-transformed FAR from the $z$-transformed HR as follows:

$$
d^{\prime}=z(H R)-z(F A R)
$$

$c$ or bias represents instead the tendency to make "yes" reports, regardless of actual stimulus presence. $c$ reflects the location of the decision criterion $k$ relative to the point of intersection between the internal distributions ( $i$ ), where the response probability is equal for signal and noise (Fig. 1 , top left). When $k=i$, the criterion is unbiased $(c=0)$. When $k>i$, the criterion is said to be conservative $(c>0)$, reflecting a bias to report "no" for both stimulus present (misses) and stimulus absent (correct rejections) trials. When $k<i$, the criterion is said to be liberal $(c<0)$, reflecting a bias to report "yes" for both stimulus present (hits) and stimulus absent (false alarms) trials. In yes/no detection paradigms, $c$ is computed as follows:

$$
c=-\frac{1}{2}(z(H R)+z(F A R))
$$

Notably, the measures of $d^{\prime}$ and $c$ depend on the assumption that the responses to the signal and to the noise are equally distributed.

Distribution-independent SDT analysis: confidence ratings were originally collected to further test the signal detection models using distributionindependent SDT measures. This is particularly relevant considering that the distribution assumption is often violated in real data, as demonstrated by Stanislaw and Todorov (1999). When the distribution assumption is violated, the measures of $d^{\prime}$ and $c$ are not independent, hindering the interpretability of the results of standard SDT analysis.

Following Kornbrot (2006), we derived distribution-independent measures of sensitivity and criterion from the area under the receiver operating characteristic $(R O C)$ curve, which is constructed by analyzing the proportion of "yes" reports in stimulus present and absent trials at different confidence levels (see Fig. 6b). Accordingly, we first constructed the $R O C$ curve and then divided the area under the ROC curve in two parts: $K_{A}$ is the area between $R O C$ curve and major diagonal below the middle ROC point; and $K_{B}$ is the area between the $R O C$ curve and the major diagonal above the middle $R O C$ point. The sensitivity measure $A_{R O C}$ represents the area between the entire $R O C$ curve and the major diagonal as follows:

$$
A_{R O C}=K_{A}+K_{B}+0.5
$$

$A_{R O C}$ values $>0.5$ correspond to above-chance performance.

The criterion measure $B_{R O C}$ is estimated by taking the logarithm of the ratio between $K_{A}$ and $K_{B}$ as follows:

$$
B_{R O C}=\log \frac{K_{A}}{K_{B}}
$$

$B_{R O C}$ values $>0$ correspond to a liberal detection bias, whereas $B_{R O C}$ values of $<0$ correspond to a conservative detection bias.

Experiment 2. We first binned trials for each task separately and then measured the HR in detection trials and the proportion of correct responses in discrimination trials for each bin and participant. The detection trials in Experiment 2 are identical to the stimulus present trials in Experiment 1 because Experiment 2 comprised only stimulus present trials.

Predictions of the signal detection models. To estimate whether oscillatory power modulates baseline excitability or response precision, we statistically tested for each time, frequency, and electrode whether behavioral estimates differed between the weakest and strongest power bins. The two models make different predictions about the relationship between prestimulus oscillatory power and the behavioral estimates measured in the two experiments.

In Experiment 1, the baseline model predicts that both the $H R$ and FAR increase in trials with weak prestimulus $\alpha$ power. According to SDT, this is equivalent to an increased tendency to make "yes" reports regardless of stimulus presence, reflected by a more liberal detection criterion (lower $c$ and higher $B_{R O C}$ ). The precision model instead predicts that trials with weak prestimulus $\alpha$ power are related to an increased $H R$ and decreased FAR. According to SDT, this is equivalent to an increase of perceptual acuity/sensitivity (higher $d^{\prime}$ and higher $A_{R O C}$ ).

In Experiment 2, according to the baseline model, decreased prestimulus $\alpha$ power is expected to increase the $H R$ in detection trials (similarly to Experiment 1) and to have no influence on the proportion of correct responses in discrimination trials. According to the precision model, decreased prestimulus $\alpha$ power is expected to increase the HR in detection trials as well as the proportion of correct responses in discrimination trials. Because Experiment 2 forbade the use of stimulus absent trials, it was not possible to further test for separate effects on sensitivity and criterion as in Experiment 1.

\section{Group-level statistical testing}

Within each subject, we first computed the difference in performance measures between the weakest and strongest power bins at each frequency, time point, and electrode. For the group-level statistical inference, we then computed the $t$ statistics of these differences against the null hypothesis that there was no difference between the bins. To determine significant effects and to correct for multiple comparisons, we used a nonparametric cluster permutation test on the absolute values of the $t$ statistics (Maris and Oostenveld, 2007) with 1000 permutations, a cluster threshold $p$ value of 0.05 , and a final significance $p$ value of 0.05 . This is the equivalent to a two-sided cluster permutation test.

In addition, to demonstrate the statistical independence of negative prestimulus effects from positive and poststimulus effects, we used a nonparametric cluster permutation test on only the negative values of the $t$ statistics of the prestimulus time window, using the same parameters as in the two-sided statistical test. This analysis is equivalent to a one-sided cluster permutation test.

Throughout the text, we use the term "effect" to refer to a significant statistical outcome, not to a causal relationship. While it appears reasonable to interpret such outcomes as indicating a causal relationship between prestimulus brain states and poststimulus performance measures, other approaches (e.g., neurostimulation) (Romei et al., 2010; Helfrich et al., 2014) are necessary to determine whether the nature of the observed effect is correlative or causal.

Fast Fourier Transform (FFT) visualization. The main analysis quantified oscillatory power on a single-trial basis and tested for resulting effects on behavior, averaged across trials within each power bin. We complemented this analysis with a simpler and more conventional approach, in which behavior ("yes" vs "no" response) was assessed on a trial-by-trial 
basis and power was averaged for each type of response. To obtain reliable power estimates, we computed power with a FFT for the timefrequency-electrode window with most sustained effect within the cluster. The FFT analysis served mainly for illustrative purposes to give the reader a more intuitive grasp of the effects identified by the binning analysis. Moreover, because the FFT is not computed by convolution, unlike wavelet analysis, results of an FFT computed over the prestimulus period cannot be influenced by poststimulus signals.

Bayes factor (BF) analysis. To provide evidence for the baseline model (Fig. 1, left), we sought to demonstrate a null effect of neural oscillations on sensitivity in Experiment 1, and on proportion of correct responses in discrimination trials of Experiment 2. However, in conventional inferential statistics, an insignificant result only indicates that the null hypothesis cannot be rejected; it does not necessarily follow that the null hypothesis is actually true. The data might also be inconclusive (e.g., due to insufficient statistical power). Thus, to directly estimate evidence for the null hypothesis, we used BF analysis (Rouder et al., 2009). We first estimated the JZS BF for the negative $t$ statistics of the difference maps, setting the prior on effect size following a Cauchy distribution with a scale factor 0.707 , as recommended by Rouder et al. (2009). BF indicates whether there is evidence for the alternative hypothesis (difference between bins, $H_{1}$ if $B F>3$ ) or for the null hypothesis (no difference between bins, $H_{0}$ if $B F<1 / 3$ ) or whether the evidence is inconclusive (if $1 / 3<B F<3$ ). We then counted for each time point the proportion of electrodes and frequencies showing evidence for $H_{1}$ and $H_{0}$.

\section{Literature review}

We conducted a systematic literature review using the search engine PubMed. Our aim was to find previous studies that examined the relationship between spontaneous/prestimulus $\alpha$ power and perception in tasks involving detection or discrimination of static visual stimuli in healthy humans. We searched in the abstracts and titles for the following key words: (spontaneous OR pre-stimulus OR prestimulus OR ongoing OR resting) AND (Alpha OR $\alpha$ OR $10 \mathrm{~Hz}$ OR $8-12 \mathrm{~Hz}$ ) AND (variability OR oscillations OR fluctuations OR activity OR rhythm OR power OR amplitude) AND (see OR perception OR performance OR detection OR discrimination OR awareness OR excitability OR percept OR percepts OR error OR errors OR hits OR misses) AND (visual OR vision OR visually). We excluded studies on unrelated topics (NOT protein, NOT tumor, etc.). The remaining studies additionally had to fulfill the following inclusion criteria: (1) direct measures of brain activity (e.g., EEG/MEG); (2) within-subject analysis; (3) perceptual reports or accuracy (e.g., not reaction times); and (4) analysis of ongoing or prestimulus $\alpha$ oscillations (e.g., not induced by transcranial magnetic stimulution or tACS) that are unaffected by experimental manipulations. Notably, this also excluded studies analyzing attention-induced lateralization or load-dependent power changes during memory tasks.

The results of the literature review are shown in Table 1. We summarized the experimental results with relational operators $(=|<|>)$, comparing prestimulus $\alpha$ power between different response types. By hit $(\mathrm{H})$ we refer to: (1) a proper hit ("yes" report in stimulus present trials) in detection tasks; (2) an aware report (Romei et al., 2008; Britz et al., 2014); or (3) a two-stimulus report in Lange et al. (2013), in which participants were required to report the number of visual stimuli perceived when two visual stimuli were presented alongside with one tactile stimulus. By miss (M), we refer to: (1) a proper miss ("no" report in stimulus present trials) in detection tasks; (2) an unaware report (Romei et al., 2008; Britz et al., 2014); or (3) a one-stimulus report (fusion effect) in Lange et al. (2013), in which participants were required to report the number of visual stimuli perceived when two visual stimuli were presented alongside with one tactile stimulus. By false alarm (FA), we refer to: (1) a proper false alarm ("yes" report in stimulus absent trials) in detection tasks; or (2) a twostimulus report (double-flash illusion) in a task where participants were required to report the number of visual stimuli perceived when one visual stimulus was presented alongside with two stimuli in another sensory modality (Lange et al., 2013; Keil et al., 2014). By correct rejection (CR), we refer to: (1) a proper correct rejection ("no" report in stimulus absent trials) in detection tasks; or (2) a one-stimulus report in Lange et al. (2013) and Keil et al. (2014).

In detection tasks (Macdonald et al., 2011; Limbach and Corballis, 2016), correct responses (C) refer to $H$ and $C R$, and incorrect responses (IN) to $\mathrm{M}$ and FA. In discrimination tasks, correct and incorrect responses refer to whether or not the stimulus is correctly discriminated. Stimulus absent trials are not required in discrimination tasks (thereby denoted by - ).

\section{Results}

\section{$\alpha$ power influences detection criterion, not sensitivity}

In Experiment 1, we recorded EEG while participants performed a visual yes/no detection task with stimulus present and absent trials (Fig. 2a), allowing us to test for an effect of $\alpha$ power on criterion (as predicted by the baseline model; Fig. 1, left) and on sensitivity (as predicted by the precision model; Fig. 1, right). The participants with above-chance performance had a mean $H R$ of 48.69\% (SEM 0.61) and mean FAR of 18.01\% (SEM 1.81; Fig. 2b, left). Mean $d^{\prime}$ and $c$ were 0.95 (SEM 0.07) and 0.51 (SEM 0.04), respectively.

We divided the trials in 5 bins based on instantaneous oscillatory power at each time, frequency, and electrode and calculated $H R, F A R$, and signal detection measures of criterion and sensitivity (distribution-dependent: $c / d$ ' and distribution-independent: $B_{R O C}\left(A_{R O C}\right)$ for each bin and participant. For the group-level statistical analysis, we used cluster permutation test to determine at which time, frequency, and electrode these measures significantly differed between the weakest and strongest power bins.

The two-sided statistical analysis of $H R$ revealed one significant cluster: $H R$ in signal present trials was increased in trials with weak prestimulus $\alpha$ and $\beta$ power $(8-22 \mathrm{~Hz})$. This cluster was significant starting from $-0.46 \mathrm{~s}$ relative to the stimulus onset (Fig. 4a). The most sustained prestimulus effect occurred at CP5, $12 \mathrm{~Hz}$ and between $-0.34 \mathrm{~s}$ and $-0.06 \mathrm{~s}$ relative to stimulus onset. Within this time-frequency-electrode window, power accounted for a difference of $9 \%$ in $H R$ (Fig. $4 c$ ). Likewise, the two-sided statistical analysis of FAR revealed one significant cluster: the FAR in signal absent trials was increased in trials with weak power $(6-30 \mathrm{~Hz})$ in a time frame around "stimulus onset" (Fig. $4 b$ ). The most sustained effect occurred at CP1, $16 \mathrm{~Hz}$ and between $-0.30 \mathrm{~s}$ and $0.30 \mathrm{~s}$ relative to "stimulus onset." In stimulus absent trials, "stimulus onset" does not refer to the onset of an actual stimulus, but to the time when a stimulus would be presented in a stimulus present trial. Within this time-frequencyelectrode window, power accounted for a difference of $13 \%$ in FAR (Fig. 4d). A FFT analysis confirmed that, compared with "no" reports, "yes" reports were associated with weaker power before stimulus onset in stimulus present trials (Fig. 4e) and at around the time when a stimulus would be presented in stimulus absent trials (Fig. 4f).

The one-sided statistical analysis yielded similar results as the two-sided test, demonstrating that negative prestimulus effects on $H R$ and FAR were statistically independent from positive and poststimulus effects (results not shown).

The analysis of signal detection measures confirmed these results (Figs. 5,6$)$. The analysis of criterion $\left(c\right.$ and $\left.B_{R O C}\right)$ indicated an increased tendency to report a stimulus regardless of actual stimulus presence (i.e., liberal detection criterion: lower $c$ and higher $B_{R O C}$ ) in trials with weak prestimulus $\alpha$ and $\beta$ power. In particular, the two-sided statistical analysis of $c$ revealed one significant cluster, comprising frequencies between 6 and $22 \mathrm{~Hz}$ and a prestimulus time window starting from $-0.61 \mathrm{~s}$ relative to stimulus onset (Fig. 5a). The most sustained prestimulus effect occurred at $\mathrm{O} 1,10 \mathrm{~Hz}$ and between $-0.46 \mathrm{~s}$ and $-0.02 \mathrm{~s}$ relative 
a

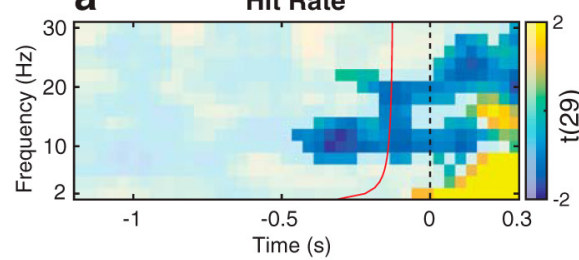

b

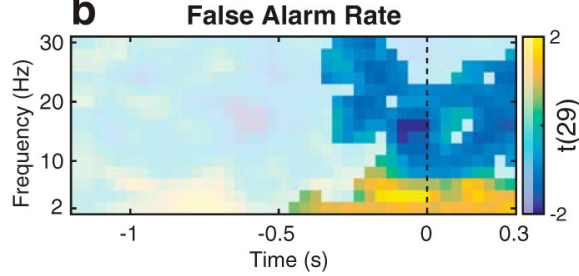

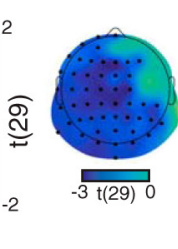

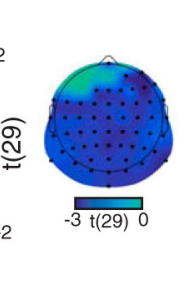

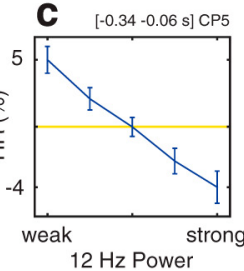

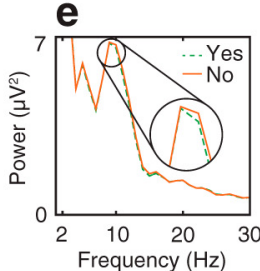

d $[-0.30+0.30 \mathrm{~s}] \mathrm{CP} 1$
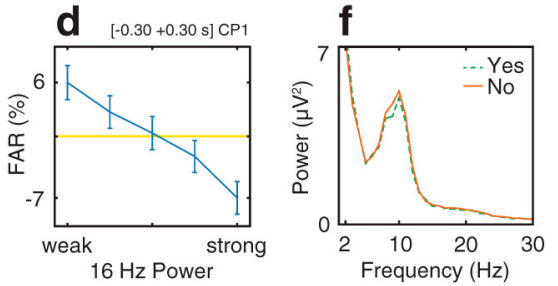

Figure 4. Relationship between oscillatory activity and the proportion of "yes" reports in Experiment 1. $\boldsymbol{a}, \boldsymbol{b}$, Group-level $t$ statistics map of the difference in hit rate (HR, $\boldsymbol{a}$ ) and false alarm rate $(F A R, \boldsymbol{b})$ between states of weak and strong oscillatory activity. The HR increases during states of weak prestimulus oscillatory activity. $\boldsymbol{a}$, The map is averaged across cluster electrodes with significant prestimulus effects. Likewise, the FAR increases during states of weak oscillatory activity. $\boldsymbol{b}$, The map is averaged across all cluster electrodes. Time 0 s indicates stimulus onset in stimulus present trials $(\boldsymbol{a})$, or the time when a stimulus would be presented in stimulus absent trials $(\boldsymbol{b})$. The red line indicates the time points before which oscillatory activity is not contaminated by poststimulus activity in stimulus-present trials $(\boldsymbol{a}) . \boldsymbol{a}, \boldsymbol{b}$, The maps are masked by a $p$ value of 0.05 using two-sided cluster permutation testing. $\boldsymbol{a}, \boldsymbol{b}$, The topographies show the negative $t$ statistics of the prestimulus $H R$ and $F A R$ cluster, respectively. Black dots represent prestimulus $(\boldsymbol{a})$ and all cluster electrodes (b) in the topographies. $\boldsymbol{c}$, $\boldsymbol{d}$, Group-average percentage change in $H R(\boldsymbol{c})$ and $F A R(\boldsymbol{d})$ in trials sorted from weak to strong oscillatory activity. A value of 0 indicates average performance across all bins (horizontal line). Error bars indicate SEM. $\boldsymbol{e}, \boldsymbol{f}$, Group-average FFT spectra computed separately for "yes" (dashed line) and "no" reports (solid line) and for stimulus present (e) and absent trials (f). Compared with "no" reports (M/CR), "yes" reports (H/FA) are related to decreased ongoing $\alpha$ power. The results visualized in $\boldsymbol{c}, \boldsymbol{e}$ and $\boldsymbol{d}, \boldsymbol{f}$ comprise a time-frequency-electrode window with most sustained effect within the prestimulus $H R$ cluster and the $F A R$ cluster, respectively.

a

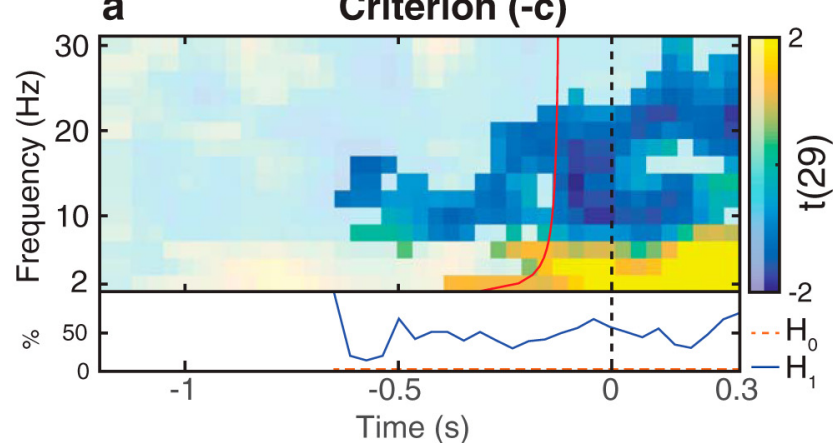

b

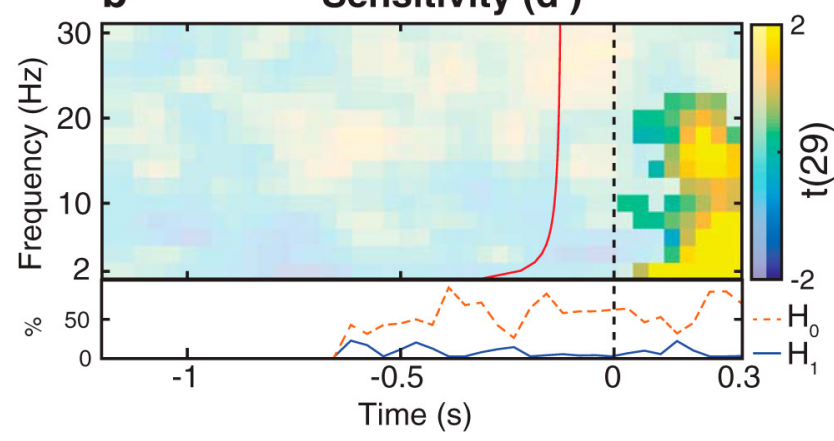

C
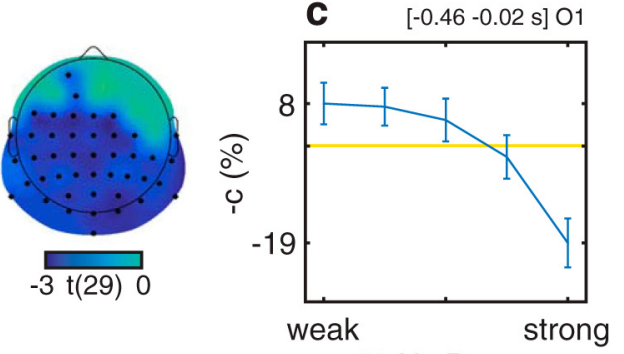

$10 \mathrm{~Hz}$ Power

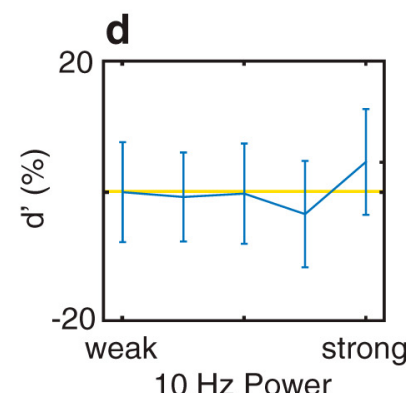

$10 \mathrm{~Hz}$ Power

Figure 5. Relationship between oscillatory activity and distribution-dependent signal detection measures in Experiment 1. $\boldsymbol{a}, \boldsymbol{b}$, Group-level $t$ statistics map of the difference in criterion $(\boldsymbol{a})$ and sensitivity $(\boldsymbol{b})$ between states of weak and strong oscillatory activity. During states of weak prestimulus oscillatory activity, stimulus presence is reported more often regardless of actual stimulus presence (higher $-c$ : more liberal criterion). $\boldsymbol{a}$, The map is averaged across cluster electrodes with significant prestimulus effects. The difference in sensitivity $\left(d^{\prime}\right)$ between states of weak and strong prestimulus oscillatory activity is not significant. $\boldsymbol{b}$, The map is averaged across cluster electrodes with significant poststimulus effects. Time 0 s indicates stimulus onset in stimulus present trials, or the time when a stimulus would be presented in stimulus absent trials. The red line indicates the time points before which oscillatory activity is not contaminated by poststimulus activity. $\boldsymbol{a}, \boldsymbol{b}$, The maps are masked by a $p$ value of 0.05 using two-sided cluster permutation testing. $\boldsymbol{a}$, The topography shows the negative $t$ statistics of the prestimulus - c cluster. Black dots represent prestimulus cluster electrodes. $\boldsymbol{a}, \boldsymbol{b}$, Bottom inset, Time course of the percentage of cluster $t$ statistics in favor of an effect of power on $-c / d^{\prime}$ ( $H_{1}$, solid line) or in favor of a null effect $\left(H_{0}\right.$, dashed line). $\boldsymbol{b}$, The BF analysis shows that there is more evidence for $H_{0}$ than for $H_{1}$, indicating that prestimulus power had a null effect on $\boldsymbol{d}^{\prime}$. $\boldsymbol{c}, \boldsymbol{d}$, Group-average percentage change in $-\boldsymbol{c}(\boldsymbol{c})$ and $\boldsymbol{d}^{\prime}(\boldsymbol{d})$ in trials sorted from weak to strong oscillatory activity. A value of 0 indicates average performance across all bins (horizontal line). Error bars indicate SEM. $\boldsymbol{c}, \boldsymbol{d}$, The results comprise a time-frequency-electrode window with the most sustained prestimulus effect within the $-\boldsymbol{c}$ cluster. 
to stimulus onset. Within this timefrequency-electrode window, (1) groupaverage criterion $(-c)$ decreased/became more conservative from weakest to strongest bin as follows: -0.4870 (SEM 0.0402 ), -0.4845 (SEM 0.0364), -0.5113 (SEM 0.0413), -0.5281 (SEM 0.0418), -0.5981 (SEM 0.0471); (2) power accounted for a difference of $27 \%$ in $c$ (Fig. $5 c$ ). The two-sided statistical analysis of $B_{R O C}$ revealed one significant cluster, comprising frequencies between 6 and $22 \mathrm{~Hz}$ and a prestimulus time window starting from -0.89 s relative to stimulus onset (Fig. 6a). The most sustained prestimulus effect occurred at $\mathrm{O} 2,10 \mathrm{~Hz}$ and between $-0.89 \mathrm{~s}$ and $-0.02 \mathrm{~s}$ relative to stimulus onset (Fig. 6b). The one-sided statistical analysis yielded similar results as the two-sided test, demonstrating that negative prestimulus effects on $c$ and $B_{R O C}$ were statistically independent from positive and poststimulus effects (results not shown).

Furthermore, the two-sided statistical analysis of sensitivity ( $d^{\prime}$ and $A_{R O C}$ ) found no significant clusters in the prestimulus time range: trials of particularly weak and strong prestimulus power did not differ in sensitivity (d', Fig. 5b,d; $A_{R O C}$, data not shown).

To substantiate these findings and to confirm that the data support a true null effect for sensitivity, as opposed to merely inconclusive evidence, we quantified for each time point the proportion of frequencies and electrodes, at which the data provided evidence for an effect $\left(\mathrm{H}_{1}\right)$ or evidence for a null effect $\left(\mathrm{H}_{0}\right)$. For time points, frequencies, and electrodes within the cluster of significant criterion effects, the proportion of data points providing evidence for a criterion effect by far outnumbered the proportion of data points supporting a null effect (c, Fig. $5 a$, bottom inset; $B_{R O C}$, data not shown). This result is expected given that the analysis was restricted to the cluster showing a significant criterion effect. However, our primary interest was to compare the strength of evidence for a criterion effect to the strength of evidence against a sensitivity effect. Because no significant prestimulus cluster of sensitivity effects was found, we conducted this comparison for the time points, frequencies, and electrodes within the criterion cluster, based on the assumption that effects on $c$ (or $B_{R O C}$ ) and $d^{\prime}$ (or $A_{R O C}$ ) would coincide in time, frequency, and space. Importantly, the proportion of data points providing evidence for a null effect on sensitivity ( $d^{\prime}$ and $A_{R O C}$ ) by far outnumbered the proportion of data points supporting a sensitivity effect ( $d^{\prime}$, Fig. $5 b$, bottom inset; $A_{R O C}$, data not shown), indicating that effects of prestimulus power on sensitivity were not merely weak or inconclusive, but absent altogether.

Together, the effect on criterion ( $c$ and $B_{R O C}$ ) and the null effect on sensitivity ( $d^{\prime}$ and $A_{R O C}$ ) are consistent with the baseline model.
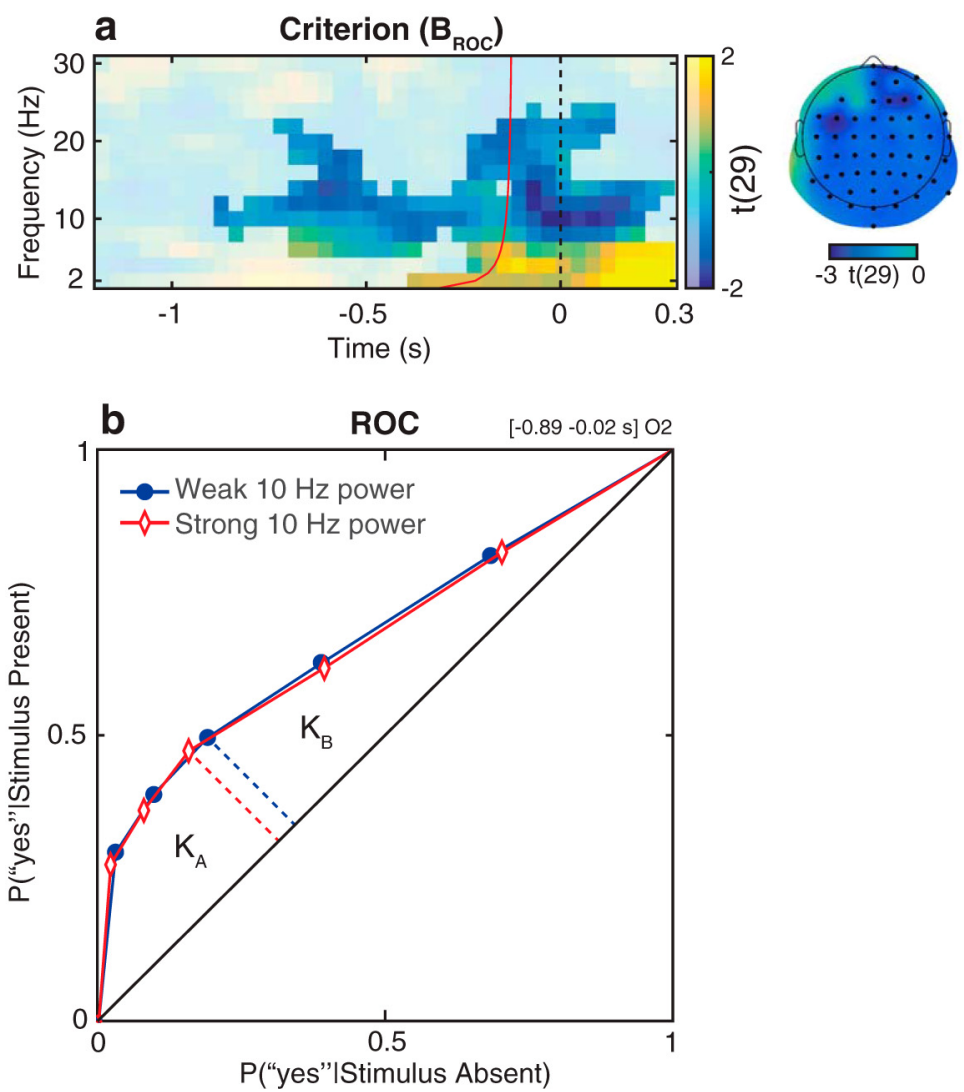

Figure 6. Relationship between oscillatory activity and the distribution-independent measure of criterion in Experiment 1. $\boldsymbol{a}$, Group-level $t$ statistics map of the difference in $B_{R O C}$ between states of weak and strong oscillatory activity. During states of weak prestimulus oscillatory activity, stimulus presence is reported more often regardless of actual stimulus presence (higher $B_{R O C}$ : more (The map is averaged across cluster electrodes with significant prestimulus effects. Time $0 \mathrm{~s}$ indicates stimulus onset in stimulus present trials, or the time when a stimulus would be presented in stimulus absent trials. The red line indicates the

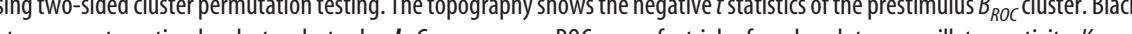
列 a increased $B_{R O C}$ (i.e., a more liberal detection criterion) during states of weak $\alpha$ power. The results visualized in $\boldsymbol{b}$ comprise a time-frequency-electrode window with the most sustained prestimulus effect within the $B_{R O C}$ cluster.

\section{$\alpha$ power influences performance in detection,} not discrimination

In Experiment 2, we compared the perceptual effects of prestimulus oscillations in detection and discrimination. Here, the baseline model predicts an effect of $\alpha$ power on detection, but not on discrimination, whereas the precision model predicts an effect of $\alpha$ power on both detection and discrimination. We recorded EEG while participants performed three tasks in a randomized block fashion: they were required to either detect the presence of nearthreshold stimuli, discriminate their location or their tilt. To enable comparison across tasks, we presented a stimulus in all trials of all tasks (Fig. 2a). An adaptive staircase procedure adjusted stimulus contrast to ensure that participants obtained a 50\% HR in the detection task and 75\% correct responses in the discrimination tasks. The resulting stimulus contrasts used for detection and location discrimination trials were not different (one-sample $t$ test: $t_{(25)}=0.94, p=0.36$ ). The stimulus contrast used for tilt discrimination trials was higher than the one used for detection trials (one-sample $t$ test: $t_{(25)}=4.26, p<0.001$ ) and location discrimination trials (one-sample $t$ test: $t_{(25)}=4.24, p<0.001$ ). There was a nonsignificant trend for an increase in contrast in the 

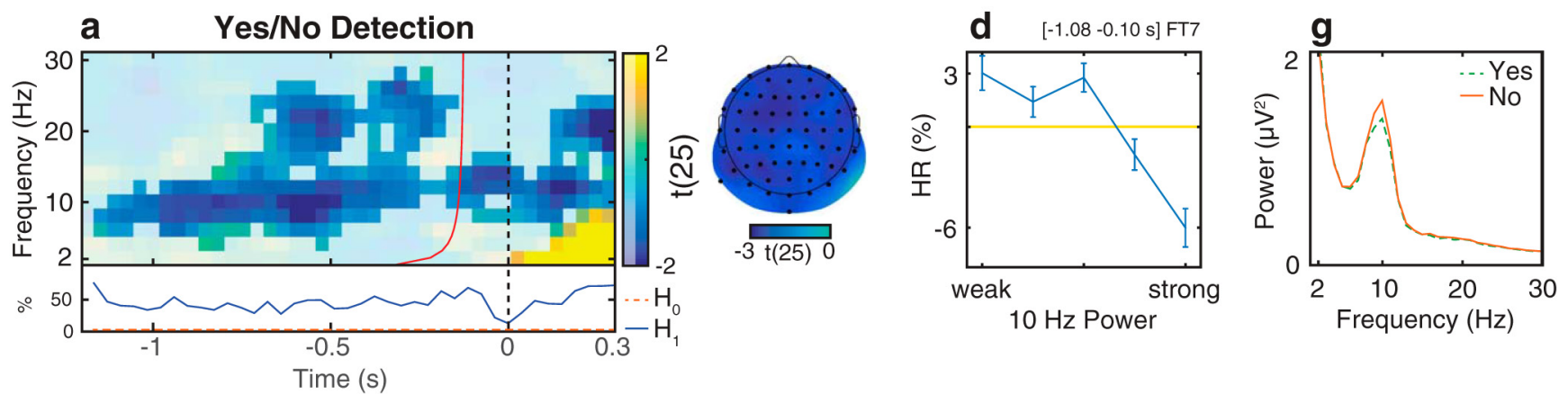

\section{b Location Discrimination}
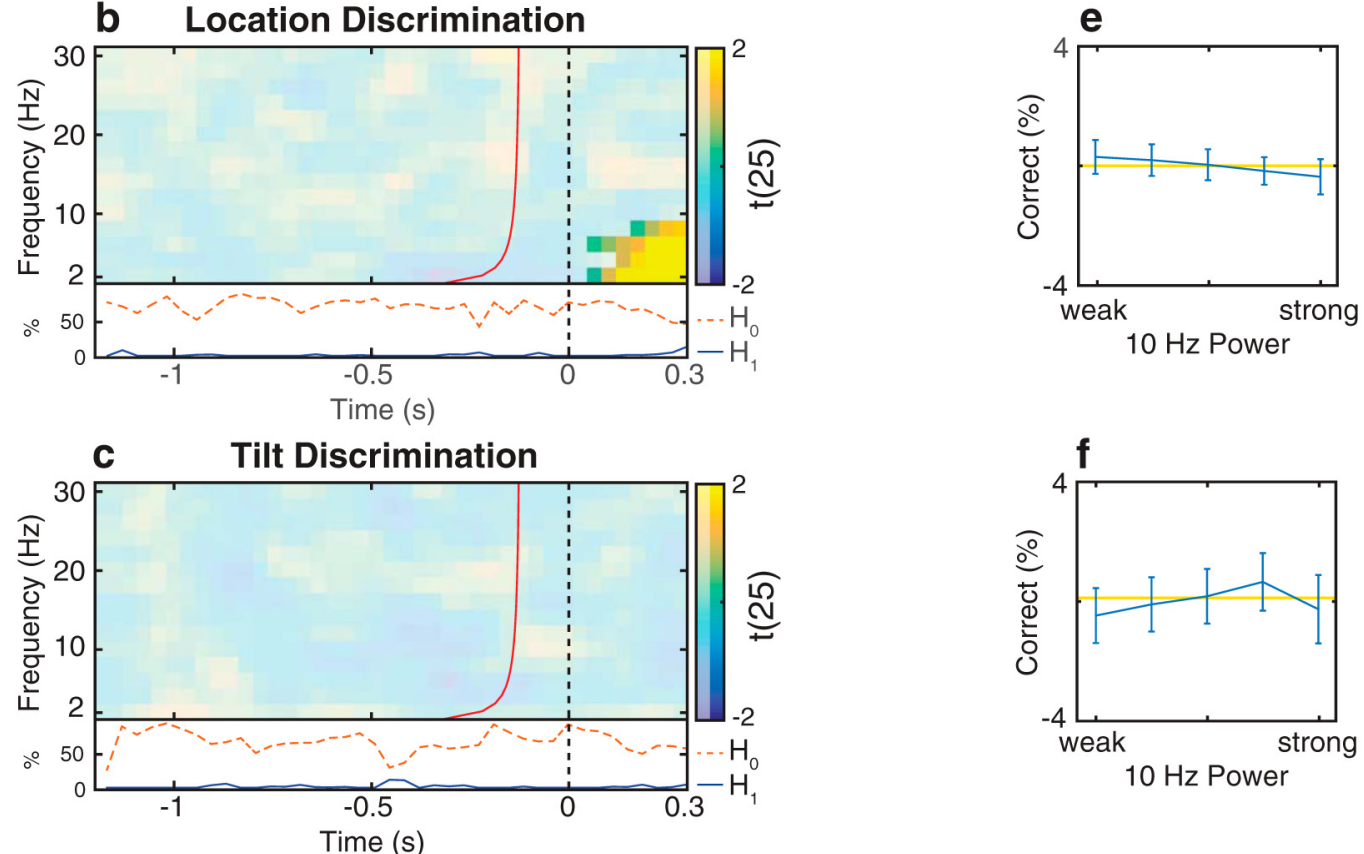

Figure 7. Relationship between oscillatory activity and detection/discrimination in Experiment 2. $\boldsymbol{a}-\boldsymbol{c}$, Group-level $t$ statistics map of the difference in hit rate (HR, $\boldsymbol{a})$ and proportion of correct discrimination responses $(\boldsymbol{b}, \boldsymbol{c})$ between states of weak and strong oscillatory activity. The $H R$ in the detection task increases during states of weak prestimulus oscillatory activity. $\boldsymbol{a}$, The map is averaged across cluster electrodes with significant prestimulus effects. By contrast, there is no difference in proportion of correct responses between states of weak and strong prestimulus oscillatory activity. $\boldsymbol{b}$, The map is averaged across cluster electrodes with significant poststimulus effects. $\boldsymbol{c}$, The map is averaged across all electrodes. Time 0 s indicates stimulus onset. The red line indicates the time points before which oscillatory activity is not contaminated by poststimulus activity. $\boldsymbol{a}-\boldsymbol{c}$, The maps are masked by a $p$ value of 0.05 using two-sided cluster permutation testing. $\boldsymbol{a}$, The topography shows the negative $t$ statistics of the prestimulus $H R$ cluster. Black dots represent prestimulus cluster electrodes. $\boldsymbol{a}-\boldsymbol{c}$, Bottom inset, Time course of the percentage of cluster $t$ statistics in favor of either an effect of power on detection/discrimination rates ( $H_{1}$, solid line) or a null effect ( $H_{0}$, dashed line). $\boldsymbol{b}, \boldsymbol{c}$, The BF analysis shows that there is more evidence for $H_{0}$ than for $H_{1}$, indicating that prestimulus power had a null effect on discrimination ability. $\boldsymbol{d}-\boldsymbol{f}$, Group-average percentage change in $H R(\boldsymbol{d})$ and proportion of correct discrimination responses $(\boldsymbol{e}, \boldsymbol{f})$ in trials sorted from weak to strong prestimulus power. 0 indicates average performance across all bins (horizontal line). Error bars indicate SEM. $\boldsymbol{g}$, Group-average FFT spectra computed for the prestimulus time window separately for "yes" (dashed line) and "no" reports (solid line) for detection trials. Compared with "no" reports (M), "yes" reports (H) are related to decreased prestimulus $\alpha$ power. $\boldsymbol{d}-\boldsymbol{g}$, The results comprise a time-frequency-electrode window with the most sustained prestimulus effect within the $H R$ cluster.

detection trials of Experiment 2 compared with the contrast used in stimulus present trials of Experiment 1 (two-sample $t$ test: $\left.t_{(54)}=1.90, p=0.063\right)$. Mean HR in the detection task was $44.99 \%$ (SEM 0.76). Mean proportion of correct responses was $73.50 \%$ (SEM 0.43) in the location discrimination task and $77.33 \%$ (SEM 0.90) in the tilt discrimination task (Fig. 2b, right).

Similar to Experiment 1, we divided the trials in 5 bins based on instantaneous oscillatory power at each time, frequency, and electrode and calculated performance measures of $H R$ in detection trials and proportion of correct responses in discrimination trials. For the group-level statistical analysis, we used a cluster permutation test to determine at which time-frequencyelectrode points these performance measures significantly differed between the weakest and strongest power bins.

The two-sided statistical analysis of $H R$ in detection trials revealed one significant cluster: $H R$ was increased in trials with weak prestimulus power $(4-28 \mathrm{~Hz})$. This cluster was significant starting from $-1.16 \mathrm{~s}$ relative to stimulus onset (Fig. $7 a$ ). The most sustained prestimulus effect occurred at FT7, $10 \mathrm{~Hz}$ and between $-1.08 \mathrm{~s}$ and $-0.10 \mathrm{~s}$ relative to stimulus onset. Within this time-frequency-electrode window, power accounted for a difference of $9 \%$ in $H R$ (Fig. 7d). A FFT analysis confirmed that "yes" reports were associated with weaker prestimulus power than "no" reports (Fig. 7g). This is consistent with the HR effect in stimulus present trials in Experiment 1 (Fig. $4 a, c, e$ ). The onesided statistical analysis yielded similar results as the two-sided test, demonstrating that negative prestimulus effect on $H R$ was statistically independent from positive and poststimulus effects (results not shown).

By contrast, the two-sided statistical analysis of the proportion of correct responses in discrimination trials revealed no significant clusters in the prestimulus time range: the proportion of 
correct responses was affected by prestimulus power in neither location (Fig. $7 b, e$ ) nor tilt discrimination trials (Fig. 7c,f).

While the data provided strong support for an effect of power on $H R$ in detection (Fig. $7 a$, bottom inset), the data within the same cluster of time, frequencies, and electrodes provided strong support for a true null effect of power on location discrimination (Fig. $7 b$, bottom inset) and tilt discrimination (Fig. $7 c$, bottom inset).

Together, the effect of prestimulus power on detection and the null effect on discrimination further support the baseline model.

\section{Literature review shows effect of $\alpha$ power on baseline- dependent measures of performance}

We conducted a systematic literature review for studies testing for an effect of prestimulus $\alpha$ oscillations on performance in visual tasks. We then categorized these studies according to whether their behavioral measures were affected (Table 1, top) or unaffected (Table 1, bottom) by a change in baseline excitability.

The majority of studies (14 of 16) using criterion-dependent measures/measures affected by a change in baseline excitability (i.e., $c$ estimates or the proportion of "yes" and "no" reports in detection tasks) found decreased $\alpha$ power preceding hits in stimulus present trials (Ergenoglu et al., 2004; Romei et al., 2008; van Dijk et al., 2008; Busch et al., 2009; Mathewson et al., 2009; Busch and VanRullen, 2010; Achim et al., 2013; Chaumon and Busch, 2014; Mathewson et al., 2014; Roberts et al., 2014; Boncompte et al., 2016; Limbach and Corballis, 2016), similar to Experiments 1 and 2, or preceding false alarms in stimulus absent trials (Limbach and Corballis, 2016), similar to Experiment 1. Two studies using a visual detection paradigm with concurrent auditory (Keil et al., 2014) or somatosensory stimuli (Lange et al., 2013) found that decreased $\alpha$ power preceded correct detections ( $\sim$ hits) but also incorrect detections ( $\sim$ false alarms) (Lange et al., 2013; Keil et al., 2014). Notably, in previous studies, stimulus absent trials were either not included in the experimental paradigm or discarded from further analysis (Table 1, Stimulus absent trials), thus precluding the estimation of SDT measures of $c$ and $d^{\prime}$ (except Limbach and Corballis, 2016). Of note, Babiloni et al. (2006) found that prestimulus source-level $\alpha$ power was reduced for misses compared with hits $(\mathrm{H}>\mathrm{M})$. In this study, the experimental paradigm required a motor response (i.e., press left/right if go stimulus is on the left/right) before the detection report. Hanslmayr et al. (2007) and Romei et al. (2008) argued that, because the detection report was delayed, this paradigm may have tested memory rather than perceptual performance, which is known to be positively related to prestimulus $\alpha$ activity. Despite this source-level effect, in the sensor space (electrode $\mathrm{Cz}$ ) $\alpha$ power was reduced in hits compared with misses $(\mathrm{H}<\mathrm{M})$, consistent with the majority of past literature. In Britz et al. (2014), $\alpha$ power did not influence awareness reports. Here, the experimental paradigm required the participants to discriminate between two stimulus alternatives (2AFC discrimination) and then to report whether or not the stimulus had been consciously perceived (aware/unaware). The analysis of this study focused on the comparison between aware and unaware reports in trials in which the stimulus was correctly discriminated. Importantly, all trials contained a stimulus. In Romei et al. (2008), the experimental paradigm required to report, after each transcranial magnetic stimulution pulse, whether or not a phosphene was perceived. This study did not include any sham trials (no stimulus absent trials). In Boncompte et al. (2016), there was a nonsignificant relationship between detection behavior and $\alpha$ power estimated in a limited time-frequency window. However, when more fre- quencies and time points were included in the analysis, prestimulus $\alpha$ and $\beta$ power was reduced in hits compared with misses $(\mathrm{H}<\mathrm{M})$ (see Boncompte et al., 2016, their Fig. 5, bottom) consistent with the majority of past literature. Because no statistical testing was performed on these extended data, we report this finding as a nonsignificant trend.

By contrast, the majority of studies (5 of 6) using criterionindependent paradigms/measures did not find a significant effect of $\alpha$ power on performance. These studies analyzed either correct responses in mAFC paradigms (similar to the 2AFC tasks in Experiment 2) (Hanslmayr et al., 2007; Wutz et al., 2014; Bays et al., 2015), correct responses in both stimulus present and absent trials in detection paradigms (Macdonald et al., 2011) or $d^{\prime}$ estimates (similar to Experiment 1) (Limbach and Corballis, 2016).

\section{Discussion}

\section{$\alpha$ power modulates baseline excitability, not response precision}

In this study, we used SDT to model the effect of spontaneous oscillatory $\alpha$ power on perception. We tested two different models, according to which $\alpha$ power affects perception either via a modulation of the baseline neural excitability or of the precision of neural responses. In Experiment 1, we analyzed the effect of $\alpha$ power on detection behavior in a yes/no detection task. We found that decreased $\alpha$ power resulted in a more liberal criterion, rather than an improved sensitivity, as predicted by the baseline model. In other words, states of decreased $\alpha$ power were related to an increased tendency to report the presence of a stimulus regardless of actual stimulus presence.

In Experiment 2, we tested a related prediction of the models by comparing the effects of $\alpha$ power on detection and discrimination. Indeed, although we replicated the finding of Experiment 1 that decreased prestimulus $\alpha$ power boosts the $H R$ in detection, we found that $\alpha$ power had no effect on location and tilt discrimination. Thus, the results of Experiment 2 further confirm the baseline model.

Together, these results strongly suggest that, contrary to previous conjectures (Ergenoglu et al., 2004; Romei et al., 2008; van Dijk et al., 2008; Roberts et al., 2014), decreased $\alpha$ power does not improve perceptual acuity but biases perception by increasing baseline excitability.

\section{Reconciling apparent inconsistencies in previous literature Visual modality}

The effect of $\alpha$ power on performance has long been interpreted as an effect on perceptual acuity. However, a number of studies were not able to find such effects; in several cases, the authors described such negative results as "surprising" and as inconsistent with previous literature (Macdonald et al., 2011; Bays et al., 2015; Strauss et al., 2015). However, our comprehensive literature review (Table 1) shows that the vast majority of published findings can be reconciled by the baseline model (Fig. 1, left). Indeed, most effects of prestimulus $\alpha$ oscillations on performance have been found by studies using detection tasks and performance measures, such as $H R$, which are affected by response criterion (Table 1, top). Thus, these studies are consistent with our results from the detection tasks of Experiments 1 and 2. By contrast, a null effect of prestimulus oscillations on performance was found in most studies using performance measures that are unaffected by baseline shifts and response criterion, such as the proportion of correct responses in mAFC paradigms/in both stimulus present and absent trials in detection paradigms or $d^{\prime}$ estimates (Table 1, bottom). Thus, these studies confirm our re- 
sults from the analysis of $d^{\prime}$ in Experiment 1 and of the discrimination tasks in Experiment 2. In sum, contrary to previous interpretations (Macdonald et al., 2011; Bays et al., 2015; Strauss et al., 2015), we argue that the pattern of positive and negative findings in previous literature is actually highly consistent and supports our proposal that spontaneous $\alpha$ oscillations modulate baseline excitability, not acuity.

\section{Nonvisual sensory modalities}

A similar, although less consistent, pattern of effects has been obtained in other sensory modalities. In auditory detection tasks, prestimulus $\alpha$ power did not affect acuity (Strauss et al., 2015) and was reduced for hits compared with misses (Herzog et al., 2014; Leske et al., 2015; for a negative finding, see Ng et al., 2012). A number of studies using somatosensory detection tasks found a similar relationship between prestimulus power and the proportion of hits and misses (Schubert et al., 2008; Weisz et al., 2014; Baumgarten et al., 2016).

Haegens et al. (2014) demonstrated that reduced $\alpha$ power in the macaque somatosensory cortex correlates with a conservative detection criterion, contrary to our finding and to the majority of reports in the visual modality (Table 1). This inconsistent finding may be explained by a number of reasons: (1) the paradigm included reward scheduling, which may have influenced the monkey's perceptual decision making; (2) the neural effects of ongoing $\alpha$ power appear to be inconsistent across the monkey's sensory-motor system (Haegens et al., 2011), suggesting different perceptual effects of $\alpha$ power in different brain areas; (3) unlike in the visual modality, the relationship between $\alpha$ power and perception may be nonlinear (Linkenkaer-Hansen et al., 2004; Zhang and Ding, 2010; Lange et al., 2013; Ai and Ro, 2014) (i.e., inverted U-shape); therefore, it is possible that the results in Haegens et al. (2014) may have resulted from sampling power only in the first half of the inverted- $U$ curve. Together, more research is required to determine whether $\alpha$ oscillations in the visual and nonvisual sensory systems show the same relationship to perceptual performance, and thus serve a similar function.

\section{Between-trials versus between-participants effects}

The present study shows that trial-by-trial fluctuations of prestimulus power affect specifically yes/no detection criterion but not discrimination performance, in line with the baseline model (Fig. 1, left). By contrast, several other studies found that differences in resting EEG power between individuals were correlated with discrimination performance (Hanslmayr et al., 2005, 2007). Recently, Limbach and Corballis (2016) found that betweensubjects variations in prestimulus $\alpha$ power were related to detection sensitivity while trial-by-trial power variations were related to detection criterion. This suggests that between-trials and between-participants effects are related to different neural and perceptual mechanisms. Unlike trial-by-trial power fluctuations, power differences between participants may indeed be related to the precision of sensory responses, as captured by the precision model (Fig. 1, right).

\section{Does $\alpha$ power modulate sensory processing or decision strategy?}

Our finding that prestimulus $\alpha$ power is related to criterion, but not sensitivity, may be seen as evidence against an effect on sensory processing, instead showing an effect on subjects' deliberate decision strategy (e.g., preferring to respond "yes" compared with "no"). However, although it is true that a change in decision strategy entails a change in measured criterion, it does not follow that any change in measured criterion, quantified according to SDT, implies a change in the subject's decision strategy (Wixted and Stretch, 2000; Witt et al., 2015). For example, a change in decision bias as accomplished by rewarding subjects for either hits or correct rejections, will affect the measured criterion. This can be represented as a change of the criterion's absolute location $k$ along the internal response axis. By contrast, a global baseline shift of excitability is expected to shift the distributions of signal and noise (i) along the internal response axis without changing the criterion's absolute position $k$ (Fig. 1, top left). This perceptual bias would affect the measured criterion without involving any change in the subject's decision strategy. Accordingly, SDT measures cannot differentiate between these two situations. Nonetheless, there is strong empirical support for the view that $\alpha$ oscillations represent a perceptual bias.

A growing number of studies have indeed demonstrated an inverse relationship between $\alpha$ power and neural excitability in sensory brain areas, as reflected in the spike-firing rate (Haegens et al., 2011), multiunit activity (van Kerkoerle et al., 2014; Becker et al., 2015), ongoing $\gamma$ power (Spaak et al., 2012) and the hemodynamic fMRI signal (Goldman et al., 2002; Becker et al., 2011; Scheeringa et al., 2011; Harvey et al., 2013; Mayhew et al., 2013).

Furthermore, numerous studies showed that $\alpha$ oscillations are involved in the gating of task-relevant perceptual information. When subjects are instructed to selectively attend to a spatial location, the strength of $\alpha$ oscillations decreases in the contralateral relative to the ipsilateral hemisphere, indicating greater excitability in the task-relevant hemisphere and greater inhibition in the task-irrelevant hemisphere (Thut et al., 2006; Busch and VanRullen, 2010; for review, see Foxe and Snyder, 2011). For example, Wyart and Tallon-Baudry (2009) concluded that attention-induced lateralization of $\alpha$ oscillations represents a shift of baseline excitability, affecting the strength of the sensory response to attended stimuli. Moreover, selective attention to a particular stimulus feature (orientation vs identity; Jokisch and Jensen, 2007) and modality (visual vs auditory; Mazaheri et al., 2014) induces a relative increase of $\alpha$ oscillations in the currently task-irrelevant areas.

Accordingly, a pattern of attention-induced decrease/increase of $\alpha$ oscillations in task-relevant/irrelevant areas can selectively amplify the strength of relevant sensory information and attenuate distracting information. By contrast, the present study focused on spontaneous fluctuations of a global or nonselective perceptual bias, which is expected to modulate the strength of both noise and signal or both stimulus alternatives. Thus, although $\alpha$ oscillations represent a perceptual bias in both cases, this bias can serve to improve task performance only when task instructions allow for its selective deployment.

In conclusion, we demonstrate that fluctuations of spontaneous $\alpha$ oscillations affect bias, rather than acuity, and that they influence performance in detection, not discrimination tasks. We propose that these oscillations bias perception by modulating baseline neural excitability, rather than the precision of sensory processing.

\section{Notes}

Supplemental material for this article is available at https://github.com/ LucaIemi/Iemi2017_JNeurosci. This material has not been peer reviewed.

\section{References}

Achim A, Bouchard J, Braun CM (2013) EEG amplitude spectra before near threshold visual presentations differentially predict detection/omission 
and short-long reaction time outcomes. Int J Psychophysiol 89:88-98. CrossRef Medline

Ai L, Ro T (2014) The phase of prestimulus alpha oscillations affects tactile perception. J Neurophysiol 111:1300-1307. CrossRef Medline

Babiloni C, Vecchio F, Bultrini A, Luca Romani G, Rossini PM (2006) Preand poststimulus alpha rhythms are related to conscious visual perception: a high-resolution EEG study. Cereb Cortex 16:1690-1700. CrossRef Medline

Baumgarten TJ, Schnitzler A, Lange J (2016) Prestimulus alpha power influences tactile temporal perceptual discrimination and confidence in decisions. Cereb Cortex 26:891-903. CrossRef Medline

Bays BC, Visscher KM, Dantec CC, Seitz AR (2015) Alpha-band EEG activity in perceptual learning. J Vis 15:1-12. CrossRef Medline

Becker R, Reinacher M, Freyer F, Villringer A, Ritter P (2011) How ongoing neuronal oscillations account for evoked fMRI variability. J Neurosci 31: 11016-11027. CrossRef Medline

Becker R, Knock S, Ritter P, Jirsa V (2015) Relating alpha power and phase to population firing and hemodynamic activity using a thalamo-cortical neural mass model. PLoS Comput Biol 11:e1004352. CrossRef Medline

Boncompte G, Villena-González M, Cosmelli D, López V (2016) Spontaneous alpha power lateralization predicts detection performance in an uncued signal detection task. PloS One 11:e0160347. CrossRef Medline

Brainard DH (1997) The Psychophysics Toolbox. Spat Vis 10:433-436. CrossRef Medline

Britz J, Diaz Hernandez L, Ro T, Michel CM (2014) EEG-microstate dependent emergence of perceptual awareness. Front Behav Neurosci 8:1-10. CrossRef Medline

Busch NA, VanRullen R (2010) Spontaneous EEG oscillations reveal periodic sampling of visual attention. Proc Natl Acad Sci U S A 107:1604816053. CrossRef Medline

Busch NA, Dubois J, VanRullen R (2009) The phase of ongoing EEG oscillations predicts visual perception. J Neurosci 29:7869-7876. CrossRef Medline

Chaumon M, Busch NA (2014) Prestimulus neural oscillations inhibit visual perception via modulation of response gain. J Cogn Neurosci 26: 2514-2529. CrossRef Medline

Chaumon M, Bishop DV, Busch NA (2015) A practical guide to the selection of independent components of the electroencephalogram for artifact correction. J Neurosci Methods 250:47-63. CrossRef Medline

Cohen MR, Maunsell JH (2009) Attention improves performance primarily by reducing interneuronal correlations. Nat Neurosci 12:1594-1600. CrossRef Medline

Delorme A, Makeig S (2004) EEGLAB: an open source toolbox for analysis of single-trial EEG dynamics including independent component analysis. J Neurosci Methods 134:9-21. CrossRef Medline

Ergenoglu T, Demiralp T, Bayraktaroglu Z, Ergen M, Beydagi H, Uresin Y (2004) Alpha rhythm of the EEG modulates visual detection performance in humans. Cogn Brain Res 20:376-383. CrossRef Medline

Foxe JJ, Snyder AC (2011) The role of alpha-band brain oscillations as a sensory suppression mechanism during selective attention. Front Psychol 2:13. CrossRef Medline

Goldman RI, Stern JM, Engel J Jr, Cohen MS (2002) Simultaneous EEG and fMRI of the alpha rhythm. Neuroreport 13:2487-2492. CrossRef Medline

Green DM, Swets JA (1966) Signal detection theory and psychophysics. New York: Wiley.

Haegens S, Nácher V, Luna R, Romo R, Jensen O (2011) $\alpha$-Oscillations in the monkey sensorimotor network influence discrimination performance by rhythmical inhibition of neuronal spiking. Proc Natl Acad Sci U S A 108:19377-19382. CrossRef Medline

Haegens S, Vázquez Y, Zainos A, Alvarez M, Jensen O, Romo R (2014) Thalamocortical rhythms during a vibrotactile detection task. Proc Natl Acad Sci U S A 111:E1797-E1805. CrossRef Medline

Hanslmayr S, Klimesch W, Sauseng P, Gruber W, Doppelmayr M, Freunberger R, Pecherstorfer T (2005) Visual discrimination performance is related to decreased alpha amplitude but increased phase locking. Neurosci Lett 375:64-68. CrossRef Medline

Hanslmayr S, Aslan A, Staudigl T, Klimesch W, Herrmann CS, Bäuml KH (2007) Prestimulus oscillations predict visual perception performance between and within subjects. Neuroimage 37:1465-1473. CrossRef Medline

Harvey BM, Vansteensel MJ, Ferrier CH, Petridou N, Zuiderbaan W, Aarnoutse EJ, Bleichner MG, Dijkerman HC, van Zandvoort MJ, Leijten FS,
Ramsey NF, Dumoulin SO (2013) Frequency specific spatial interactions in human electrocorticography: V1 alpha oscillations reflect surround suppression. Neuroimage 65:424-432. CrossRef Medline

Helfrich RF, Schneider TR, Rach S, Trautmann-Lengsfeld SA, Engel AK, Herrmann CS (2014) Entrainment of brain oscillations by transcranial alternating current stimulation. Curr Biol 24:333-339. CrossRef Medline

Herzog L, Salehi K, Bohon KS, Wiest MC (2014) Prestimulus frontalparietal coherence predicts auditory detection performance in rats. J Neurophysiol 111:1986-2000. CrossRef Medline

Jokisch D, Jensen O (2007) Modulation of gamma and alpha activity during a working memory task engaging the dorsal or ventral stream. J Neurosci 27:3244-3251. CrossRef Medline

Keil J, Müller N, Hartmann T, Weisz N (2014) Prestimulus beta power and phase synchrony influence the sound-induced flash illusion. Cereb Cortex 24:1278-1288. CrossRef Medline

Kornbrot DE (2006) Signal detection theory, the approach of choice: model-based and distribution-free measures and evaluation. Percept Psychophys 68:393-414. CrossRef Medline

Lange J, Halacz J, van Dijk H, Kahlbrock N, Schnitzler A (2012) Fluctuations of prestimulus oscillatory power predict subjective perception of tactile simultaneity. Cereb Cortex 22:2564-2574. CrossRef Medline

Lange J, Oostenveld R, Fries P (2013) Reduced occipital alpha power indexes enhanced excitability rather than improved visual perception. J Neurosci 33:3212-3220. CrossRef Medline

Leske S, Ruhnau P, Frey J, Lithari C, Müller N, Hartmann T, Weisz N (2015) Prestimulus network integration of auditory cortex predisposes nearthreshold perception independently of local excitability. Cereb Cortex 25:4898-4907. CrossRef Medline

Limbach K, Corballis PM (2016) Prestimulus alpha power influences response criterion in a detection task. Psychophysiology 53:1154-1164. CrossRef Medline

Linkenkaer-Hansen K, Nikulin VV, Palva S, Ilmoniemi RJ, Palva JM (2004) Prestimulus oscillations enhance psychophysical performance in humans. J Neurosci 24:10186-10190. CrossRef Medline

Lou B, Li Y, Philiastides MG, Sajda P (2014) Prestimulus alpha power predicts fidelity of sensory encoding in perceptual decision making. Neuroimage 87:242-251. CrossRef Medline

Macdonald JS, Mathan S, Yeung N (2011) Trial-by-trial variations in subjective attentional state are reflected in ongoing prestimulus EEG alpha oscillations. Front Psychol 2:82. CrossRef Medline

Maris E, Oostenveld R (2007) Nonparametric statistical testing of EEG- and MEG-data. J Neurosci Methods 164:177-190. CrossRef Medline

Mathewson KE, Gratton G, Fabiani M, Beck DM, Ro T (2009) To see or not to see: pre-stimulus alpha phase predicts visual awareness. J Neurosci 29:2725-2732. CrossRef Medline

Mathewson KE, Beck DM, Ro T, Maclin EL, Low KA, Fabiani M, Gratton G (2014) Dynamics of alpha control: preparatory suppression of posterior alpha oscillations by frontal modulators revealed with combined EEG and event-related optical signal (EROS). J Cogn Neurosci 26:2400-2415. CrossRef Medline

Mayhew SD, Ostwald D, Porcaro C, Bagshaw AP (2013) Spontaneous EEG alpha oscillation interacts with positive and negative BOLD responses in the visual-auditory cortices and default-mode network. Neuroimage 76 : 362-372. CrossRef Medline

Mazaheri A, van Schouwenburg MR, Dimitrijevic A, Denys D, Cools R, Jensen O (2014) Region-specific modulations in oscillatory alpha activity serve to facilitate processing in the visual and auditory modalities. Neuroimage 87:356-362. CrossRef Medline

Mazaheri A, Jensen O (2010) Rhythmic pulsing: linking ongoing brain activity with evoked responses. Front Hum Neurosci 4:177. CrossRef Medline

Mitchell JF, Sundberg KA, Reynolds JH (2009) Spatial attention decorrelates intrinsic activity fluctuations in macaque area V4. Neuron 63:879888. CrossRef Medline

Ng BS, Schroeder T, Kayser C (2012) A precluding but not ensuring role of entrained low-frequency oscillations for auditory perception. J Neurosci 32:12268-12276. CrossRef Medline

PelliDG (1997) The VideoToolbox software for visual psychophysics: transforming numbers into movies. Spat Vis 10:437-442. CrossRef Medline

Roberts DM, Fedota JR, Buzzell GA, Parasuraman R, McDonald CG (2014) Prestimulus oscillations in the alpha band of the EEG are modulated by the difficulty of feature discrimination and predict activation of a sensory discrimination process. J Cogn Neurosci 26:1615-1628. CrossRef Medline 
Romei V, Brodbeck V, Michel C, Amedi A, Pascual-Leone A, Thut G (2008) Spontaneous fluctuations in posterior $\alpha$-band EEG activity reflect variability in excitability of human visual areas. Cereb Cortex 18:2010-2018. CrossRef Medline

Romei V, Gross J, Thut G (2010) On the role of prestimulus alpha rhythms over occipito-parietal areas in visual input regulation: correlation or causation? J Neurosci 30:8692-8697. CrossRef Medline

Rouder JN, Speckman PL, Sun D, Morey RD, Iverson G (2009) Bayesian t tests for accepting and rejecting the null hypothesis. Psychon Bull Rev 16:225-237. CrossRef Medline

Scheeringa R, Mazaheri A, Bojak I, Norris DG, Kleinschmidt A (2011) Modulation of visually evoked cortical fMRI responses by phase of ongoing occipital alpha oscillations. J Neurosci 31:3813-3820. CrossRef Medline

Schubert R, Haufe S, Blankenburg F, Villringer A, Curio G (2008) Now you'll feel it-now you won't: EEG rhythms predict the effectiveness of perceptual masking. J Cogn Neurosci 21:2407-2419. CrossRef Medline

Spaak E, Bonnefond M, Maier A, Leopold DA, Jensen O (2012) Layerspecific entrainment of gamma-band neural activity by the alpha rhythm in monkey visual cortex. Curr Biol 22:2313-2318. CrossRef Medline

Stanislaw H, Todorov N (1999) Calculation of signal detection theory measures. Behav Res Methods Instrum Comput 31:137-149. CrossRef Medline

Strauß A, Henry MJ, Scharinger M, Obleser J (2015) Alpha phase determines successful lexical decision in noise. J Neurosci 35:3256-3262. CrossRef Medline

Tallon-Baudry C, Bertrand O, Delpuech C, Pernier J (1996) Stimulus specificity of phase-locked and non-phase-locked $40 \mathrm{~Hz}$ visual responses in human. J Neurosci 16:4240-4249. Medline

Thut G, Nietzel A, Brandt SA, Pascual-Leone A (2006) Alpha-band electroencephalographic activity over occipital cortex indexes visuospatial attention bias and predicts visual target detection. J Neurosci 26:9494-9502. CrossRef Medline van Dijk H, Schoffelen JM, Oostenveld R, Jensen O (2008) Prestimulus oscillatory activity in the alpha band predicts visual discrimination ability. J Neurosci 28:1816-1823. CrossRef Medline

van Kerkoerle T, Self MW, Dagnino B, Gariel-Mathis MA, Poort J, van der Togt C, Roelfsema PR (2014) Alpha and gamma oscillations characterize feedback and feedforward processing in monkey visual cortex. Proc Natl Acad Sci U S A 111:14332-14341. CrossRef Medline

Watson AB, Pelli DG (1983) QUEST: a Bayesian adaptive psychometric method. Percept Psychophys 33:113-120. CrossRef Medline

Weisz N, Wühle A, Monittola G, Demarchi G, Frey J, Popov T, Braun C (2014) Prestimulus oscillatory power and connectivity patterns predispose conscious somatosensory perception. Proc Natl Acad Sci U S A 111: E417-E425. CrossRef Medline

Widmann A, Schröger E, Maess B (2015) Digital filter design for electrophysiological data: a practical approach. J Neurosci Methods 250:34-46. CrossRef Medline

Witt JK, Taylor JE, Sugovic M, Wixted JT (2015) Signal detection measures cannot distinguish perceptual biases from response biases. Perception 44:289-300. CrossRef Medline

Wixted JT, Stretch V (2000) The case against a criterion-shift account of false memory. Psychol Rev 107:368-376. CrossRef Medline

Wutz A, Weisz N, Braun C, Melcher D (2014) Temporal windows in visual processing: 'prestimulus brain state' and 'poststimulus phase reset' segregate visual transients on different temporal scales. J Neurosci 34:15541565. CrossRef Medline

Wyart V, Tallon-Baudry C (2009) How ongoing fluctuations in human visual cortex predict perceptual awareness: baseline shift versus decision bias. J Neurosci 29:8715-8725. CrossRef Medline

Zhang Y, Ding M (2010) Detection of a weak somatosensory stimulus: role of the prestimulus mu rhythm and its top-down modulation. J Cogn Neurosci 22:307-322. CrossRef Medline 